\title{
RESPONSABILIDADE AMBIENTAL E GERENCIAMENTO DE RESULTADOS EM EMPRESAS DE GOVERNANÇA CORPORATIVA DA BM\&FBOVESPA
}

\section{ENVIRONMENTAL RESPONSIBILITY AND EARNING MANAGEMENT IN COMPANIES OF CORPORATE GOVERNANCE IN BM\&F BOVESPA}

\author{
Ilse Maria Beuren*E-mail: ilse.beuren@gmail.com \\ Moacir Manoel Rodrigues Junior** E-mail: moacir ro@hotmail.com \\ *Universidade Federal do Paraná (UFPR), Curitiba, PR \\ ** Universidade Regional de Blumenau (FURB), Blumenau, SC
}

\begin{abstract}
Resumo: Este estudo objetiva analisar se empresas que possuem a característica de serem socioambientalmente responsáveis tendem a gerenciar mais seus resultados do que as sem essa característica. Fundamentado na pesquisa de Heltzer (2011), realizou-se pesquisa descritiva com abordagem quantitativa, por meio de análise documental, em empresas dos níveis de governança corporativa da BM\&FBovespa, diferenciando-as pela existência ou não de atividades ambientais. Para o cálculo do índice de gerenciamento de resultados utilizaram-se quatro modelos, sendo um o modelo Jones original e os outros três modelos modificados do primeiro. Os resultados apontaram que empresas que são ambientalmente responsáveis possuem um índice de gerenciamento de resultados negativo maior que empresas sem esta característica. Como conclusão tem-se que as atividades de responsabilidade ambiental influenciaram significativamente o resultado das empresas socioambientalmente responsáveis pesquisadas, o que denota gerenciamento de resultados mediante decisões operacionais, mesmo que de modo não consciente pelos gestores.
\end{abstract}

Palavras-chave: Responsabilidade ambiental. Gerenciamento de resultados. Decisões operacionais dos gestores. Empresas de governança corporativa. Socioambientalmente responsáveis

\begin{abstract}
The goal of this study is to examine whether companies that have the characteristic of being socially and environmentally responsible tend to manage more their earning than those without this characteristic. Based on Heltzer's (2011) research, a descriptive research with quantitative approach was carried out, through documentary analysis, in companies of corporate governance levels in BM \& F Bovespa, differentiating them by the existence of environmental activities or not. Four models were used to calculate the index of earnings management, being one the original Jones model and the other three modified models from the first. The results showed that companies that are environmentally responsible have an index greater negative earnings management than companies without this characteristic. It was concluded that environmental responsibility activities have significantly influenced the outcome of the socially and environmentally responsible companies surveyed, which indicates earnings management through operational decisions, even if not consciously, by management.
\end{abstract}

Keywords: Environmental responsibility. Earning management. Managers' operational decisions. Companies of corporate governance. Socially and environmentally responsible. 


\section{INTRODUÇÃO}

O poder conferido ao cargo que os gestores ocupam pode leva-los a tomar decisões que vão de encontro com os interesses dos acionistas ou dos proprietários das empresas. Estas atitudes muitas vezes são tomadas para que os dados econômico-financeiros apresentados nas demonstrações contábeis não venham a prejudicar o gestor no cargo que ocupa na empresa ou motivadas por desempenho em desacordo com o que foi projetado para o período.

Chih, Shen e Kang (2008) descrevem que os escândalos que envolveram grandes empresas norte americanas, como a Enron, Tyco, BMY, WorldCom, Xerox e Merck, foram gerados pelo acumulo de poder nas mãos dos gestores. Para estes autores, o poder concentrado pelos gestores thes deu a oportunidade de gerenciar seus resultados, a fim de maximizar seus ganhos na empresa. Segundo os autores, o comentário que surgiu quando ocorreram os escândalos, foi que estes eram provocados pela deterioração de aspectos morais ou a falha da política de responsabilidade social da empresa.

Estas fraudes denunciadas nas demonstrações contábeis das empresas citadas implicaram em providências pelos órgãos reguladores da contabilidade para inibir procedimentos dessa natureza, como também oportunizaram a realização de pesquisas sobre as fragilidades organizacionais que permitiram tais atitudes. Holanda et al. (2012) descrevem que "a responsabilidade social corporativa é tida como uma forma de relação entre a empresa e a sociedade, pois visa à satisfação dos funcionários e o bem-estar da sociedade". Os autores complementam que a empresa necessita possuir compromisso com seus empregados e a comunidade.

O gerenciamento de resultados, por sua vez, pode ser visto como um meio que os gestores utilizam para valorizar os aspectos que lhes são favoráveis (HEALY; WAHLEN, 1999). Esta afirmação corrobora com o que defendem Martinez e Cardoso (2009), quando afirmam que os gestores buscam sempre evidenciar melhores resultados, manipulando os resultados contábeis, mesmo que para fins operacionais. Este fato se dá segundo os autores pelo gerenciamento de resultados contábeis mediante as decisões operacionais. Healy e Wahlen (1999) defendem que 
esta forma de gerenciamento de resultados é presente quando da adoção de novas estratégias para a empresa, ou na tomada de decisão para a contratação de novos empregados, bem como a adoção de uma política diferenciada para a empresa.

Heltzer (2011, p. 66) descreve que, quando a adoção de alguma política traz retorno para a empresa, e esta empresa não atende a expectativa estabelecida, os gestores tendem a gerenciar seus resultados para que os stakeholders vejam que a atividade não traz prejuízo. Isto ocorre muitas vezes quando a empresa adota atividades relacionadas às questões sociais e ambientais, o retorno destas atividades pode não ser diretamente financeiro, mas pode afetar no desempenho da empresa.

$\mathrm{Na}$ linha descrita por Heltzer (2011), que investigou empresas norte americanas, este estudo busca responder a seguinte questão de pesquisa: Existe diferença no gerenciamento de resultados mediante decisões operacionais de empresas listadas nos níveis de governança corporativa da BM\&FBovespa que possuem responsabilidade socioambiental e as sem esta característica? Assim o objetivo do estudo é analisar se empresas que possuem a característica de serem socioambientalmente responsáveis tendem a gerenciar mais seus resultados do que as sem essa característica.

Por se tratar da replicação da pesquisa de Heltzer (2011) em empresas brasileiras, cabe reafirmar a justificativa dada pelo autor no estudo original. A "contribuição deste trabalho com a literatura de Gerenciamento de Resultados está em expandir a base de conhecimento existente em relação a situações em que há um aumento significativo no Gerenciamento de Resultados" (HELTZER, 2011, p. 66). O autor ainda afirma que se pode partir do pressuposto que empresas que possuem alguma característica social e ou ambiental tendem a gerenciar mais seus resultados. A contribuição também está em discutir por que o gerenciamento ocorre em empresas com estas características.

A existência de uma relação positiva entre o gerenciamento de resultados e as atividades de responsabilidade social das empresas é defendia por Hall e Stammerjohan (1997), Han e Wang (1998), Prior, Surruca e Tribó (2008), entre outros. Em contraposição aos achados que afirmam relação positiva entre 
gerenciamento de resultados e responsabilidade social das empresas, o próprio trabalho de Heltzer (2011) apresenta os resultados da não existência de relação. Este fato levou o autor a considerar que tratava-se de práticas institucionalizadas e que estas eram feitas em conformidade com o interesse dos acionistas. Portanto, justifica-se a reaplicação desse estudo em outras realidades, como o que se propõe.

\section{RESPONSABILIDADE SOCIAL E AMBIENTAL}

Responsabilidade social empresarial vem à tona nas discussões teóricas, inicialmente na década de 1950, quando se questionou a responsabilidade de empresas que em seu meio de produção consomem recursos naturais, gerando poluentes eliminados diretamente no ecossistema. $O$ trabalho pioneiro nesta linha foi o de Howard R. Bowen, que considerou na época que grandes empresas exerciam fortes impactos na vida das pessoas, e questionava se a empresa havia se dado conta deste fato (RIBEIRO et al. 2009). Para Santana, Périco e Rebelatto (2006, p. 125), esta definição vem evoluindo com o passar dos anos.

Carroll (1999) defende que, por responsabilidade social das empresas subentende-se a responsabilidade ambiental inclusa, é fruto da associação de quatro princípios básicos: responsabilidade econômica, responsabilidades legais, responsabilidades éticas e responsabilidades discricionárias. Neste contexto é possível descrever, segundo Ashley (2003, p, 6), responsabilidade social como um conjunto de atividades que as empresas devem possuir para com a sociedade de modo geral, ou para com uma comunidade em específico, que promovam a melhoria do bem estar social. Estas atitudes, segundo os autores, devem vir de forma proativa das organizações.

Donaire (1999, p. 200) descreve que a responsabilidade social "assume diversas formas, entre as quais se incluem proteção ambiental, projetos filantrópicos e educacionais, planejamento da comunidade, equidade nas oportunidades de emprego, serviços sociais em geral, de conformidade com o interesse público". Isto corrobora com os princípios estabelecidos por Carroll (1999), incluindo também a questão ambiental na responsabilidade social. 
Com esta postura descrita por Donaire (1999), de que as empresas para serem socialmente responsáveis devem desempenhar tarefas sociais, é que se pode correlacionar o gerenciamento de resultados ao nível operacional, como descreve Roychowdhury (2006). As atividades de responsabilidade social estão inclusas como maneiras que os gestores encontram para gerenciar seus resultados. A idéia está atrelada também ao que afirmam Santana, Périco e Rebelatto (2006), que empresas que possuem característica de responsabilidade social possuem maior visibilidade na mídia, o que pode coadunar com a adoção da prática de gerenciamento de resultados por parte dos gestores.

O viés mais explorado em pesquisas nacionais sobre a responsabilidade social das empresas, segundo Santana, Périco e Rebelatto (2006), é a avaliação do desempenho. Segundo os autores, a partir do ano de 2000, pesquisas sobre esta característica no Brasil passaram a se focalizar em avaliar se o desempenho de empresas que adotam características sócio ambientais são afetadas.

Silva et al. (2013) avaliaram os resultados da Petrobrás confrontando-os com os indicadores de Responsabilidade Social da Empresa. A relação foi medida por meio da comparação do faturamento líquido da empresa e os investimentos em atividades socioambientais presentes no balanço social da empresa. Neste estudo constaram diferenciação de investimentos sociais e ambientais, e os resultados obtidos mostram maior preocupação com indicadores sociais.

Hall e Stammerjohan (1997) e Han e Wang (1998) estudaram a relação entre o gerenciamento de resultados em empresas que adotaram a característica de ser ambientalmente responsáveis. Ambas as pesquisas apresentam casos de empresas que utilizaram-se de atividades ambientais para aumentar seus custos políticos e assim gerenciar seus resultados. Admite-se que com custos políticos elevados, as empresas tendem a gastar mais com campanhas para manter sua reputação na sociedade.

Hall e Stammerjohan (1997) testaram as preocupações ambientais de empresas americanas de petróleo, o que resultou na condição que os gestores utilizaram-se da elevação do custo público como forma de gerenciar os resultados da empresa. Han e Wang (1998) avaliaram empresas do setor petroquímico 
americano, e destacaram que estas empresas gerenciaram seus resultados durante a crise do Golfo para mostrar que, por conta de suas atividades, essas estavam em uma situação de desvantagem, justificando assim um aumento brusco nos preços dos produtos.

Tomando como um dos viéses da responsabilidade social, que é a ética, Chih, Shen, Kang (2007) destacaram em seu estudo que os problemas com crises em empresas americanas, como a Eron, foram provocados pelo gerenciamento excessivo de seus resultados. O que fere a responsabilidade social quando se adota como viés a ética da empresa. O estudo analisou a qualidade das demonstrações contábeis com esta característica em comparação com empresas sem responsabilidade, e observaram que empresas sem responsabilidade social declarada possuem maior qualidade nas suas demonstrações contábeis, consequentemente menor gerenciamento de resultados.

Outro estudo que faz uma comparação entre responsabilidade social e gerenciamento de resultados é o de Prior, Surroca e Tribo (2008). Os autores compararam empresas de 26 países, sendo que algumas possuíam atividades sociais e outras não. Os resultados da pesquisa revelaram que quanto maior a responsabilidade social das empresas maior é o gerenciamento de resultados, pois os gestores tenderam a declarar maiores custos na manutenção destas atividades sociais. A pesquisa também descreveu uma relação inversamente proporcional entre o nível de responsabilidade ambiental e o desempenho da empresa.

Para Tachizawa (2009), as práticas de atividades socioambientais se diferem quando levado em consideração o setor de atuação das empresas. A distinção entre os setores de atuação leva a considerar diferentes projetos de atuação das empresas, o que justifica a análise por tipo de projeto realizada por Heltzer (2011).

Diante do exposto, este estudo procura verificar se os achados das pesquisas relatadas acima se verificam quando se trata de empresas brasileiras. Neste estudo pretende-se comparar os resultados obtidos por Heltzer (2011), o que é possível justamente por se configurar em replicação da pesquisa citada em um cenário diferente. 


\section{CARACTERÍSTICAS DA PESQUISA DE HELTZER (2011)}

O artigo de Heltzer (2011), sob o título de "The asymmetric relationship between corporate environmental responsibility and earnings managements: evidence from the United States", publicado no periódico internacional Management, Auditing Journal, foi escolhido porque descreve as atividades de responsabilidade ambiental como uma forma de os gestores gerenciarem seus resultados, indo de encontro com o interesse dos acionistas. Objetivou "analisar as relações entre os ganhos de gestão (gerenciamento de resultados) e subamostras de responsabilidade ambiental das empresas". O pressuposto foi que, ao desenvolver atividades de responsabilidade ambiental nas empresas, estas gerariam maiores gastos, sendo uma forma de diminuir o lucro destinado aos acionistas.

O estudo apresenta uma abordagem diferenciada tanto para o gerenciamento de resultados, quanto para as atividades ambientais. No que diz respeito à abordagem do gerenciamento de resultados, o autor considera que o gerenciamento é feito por meio de atividades que o gestor desenvolve dentro de uma organização e estas atividades vão de encontro com o interesse dos acionistas de aumentar seus lucros. Na abordagem da responsabilidade ambiental, o autor comenta sobre a possibilidade de o gestor desenvolver estas atividades, mais que diminuir seu resultado, estará aumentando seus gastos. Estas são as contribuições do estudo, em que busca vincular, as práticas ambientais das empresas com o modo que seus gestores as utilizam, direta ou indiretamente, para gerenciar seus resultados.

A análise compreendeu 2.171 empresas listadas no Kinder Lydenberg and Domini (KLD), utilizando as informações mais recentes disponíveis. Esta base de dados disponibiliza a relação das atividades de responsabilidade ambiental de duas formas. A primeira é pela força ambiental, que consiste de atividades diretamente desempenhadas no combate e prevenção da depredação do meio ambiente, subdivididas em seis categorias: (1) produtos e serviços benéficos; (2) prevenção da poluição; (3) reciclagem; (4) energia limpa; (5) sistema de gestão ambiental; e (6) outras forças. A segunda compreende as preocupações ambientais, que se constituem de atividades externas as empresas, subdivididas em sete categorias: (1) 
resíduos perigosos; (2) problemas de regulamentação; (3) redutores de ozônio; (4) emissões significativas; (5) produtos químicos agrícolas; (6) alterações climáticas; e (7) outras preocupações.

Os dados foram dispostos de forma dicotômica, zero para não possui e 1 para possui. A análise foi dividida de forma a responder cinco questões elaboradas pelo autor. São elas:

RQ1.Empresas com responsabilidade ambiental exibem diferença estatisticamente significativa no gerenciamento de resultados se comparadas com empresas sem esta característica?

RQ2.Empresas com forças ambientais exibem diferença estatisticamente significativa no gerenciamento de resultados se comparadas com empresas sem esta característica?

RQ3.Empresas com preocupação ambiental exibem diferença estatisticamente significativa no gerenciamento de resultados se comparadas com empresas que não possuem preocupação ambiental?

RQ4.Empresas com múltiplos níveis de forças ambientais exibem estatisticamente diferentes níveis de gerenciamento de resultados do que as empresas com uma única força ambiental?

RQ5.Empresas com múltiplos níveis de preocupações ambientais exibem estatisticamente diferentes níveis de gerenciamento de resultados do que as empresas com uma única preocupação ambiental?

Os resultados descritos pelo autor mostram que, com referência as questões $R Q 1$ e RQ4, a relação entre responsabilidade social e gerenciamento de resultados, pela literatura apresentada pelo autor esta relação segue uma abordagem institucional. Este fato é corroborado pelos resultados, pois não houve diferença no gerenciamento entre empresas com responsabilidade social e empresas sem esta característica (RQ1), e o aumento das responsabilidades não interfere no nível do gerenciamento de resultados.

Considerando as questões RQ2, RQ3 e RQ5 separadamente, os resultados apóiam a hipótese de evitar a miopia. Os achados também apontam para uma relação negativa entre as preocupações ambientais e o gerenciamento de 
resultados, o que indica que quando diminui o número de elementos de preocupação ambiental o índice de gerenciamento de resultados irá aumentar. $O$ autor afirma que, "[...] os resultados relativos às forças ambientais sugerem que o respeito ao meio ambiente é devido a fatores institucionais, que estão relacionadas com o gerenciamento de resultados". Por outro lado o autor confirma que, "[...] os resultados relativos às questões ambientais sugerem que as empresas que desrespeitam o meio ambiente são mais propensos a esconder ganhos desfavoráveis, vis -à- vis outras empresas da amostra".

Estes são, portanto, os aspectos descritos no artigo que se replicam nesta pesquisa. Assim, os procedimentos de pesquisa para este estudo são idênticos aos relatados neste tópico, com o diferencial de ser voltado para empresas brasileiras. Na seqüência descreve-se detalhadamente o processo de coleta e análise de dados desta pesquisa, com vistas na mensuração do gerenciamento de resultados.

\section{GERENCIAMENTO DE RESULTADOS}

Diferentes formas de gerenciamento de resultados são abordadas na literatura relacionada ao tema. A forma mais comum, segundo Martinez e Cardoso (2009), é a do gerenciamento de resultados por meio de escolhas contábeis. Outra maneira encontrada para o gerenciamento de resultados é por meio das decisões operacionais, que os autores afirmam ser uma forma que dificilmente é percebida em análises.

Como na pesquisa que consubstancia este estudo foi considerado o gerenciamento de resultados operacionais, discorre-se mais detalhadamente sobre essa modalidade, para fundamentar a mensuração e análise dos dados.

\subsection{Gerenciamento de Resultados por Decisões Operacionais}

Uma definição de gerenciamento de resultados trazida por Healy e Wahlen (1999) afirma que é a atividade dos gestores de descreverem seus resultados de forma a não revelar a real situação para um determinado grupo de stakeholders, 
quando existe conflito de interesses. Esta definição mostra a abrangência do tema, pois para grupos diferentes de usuários da informação contábil se tem objetivos diferentes de gerenciar os resultados.

Roychowdhury (2006, p. 337) concorda com a definição acima e esclarece que o gerenciamento de resultados não está na manipulação da informação contábil propriamente dita, mas está inserido nas atividades operacionais da empresa. Segundo a autora, em muitos casos os gestores aumentam exponencialmente a produção para mostrar a redução no custo de produção, bem como "[...] a aceleração das vendas, as alterações nos horários de transporte, e o atraso de pesquisa e desenvolvimento (R\&D) e manutenção de despesas como métodos de gerenciamento de resultados disponíveis para os gestores".

Martinez e Cardoso(2009) afirmam que o gerenciamento de resultados realizado por meio das decisões operacionais é muitas vezes realizado para burlar a regulamentação contábil. Esta discussão traz o gerenciamento de resultado como sendo lícito, desta forma não se caracterizando como fraude. Burgstahler e Dichev (1997) destacam que quando uma empresa pretende diminuir seu lucro, para não distribuir tanto aos acionistas, os gestores tendem a aumentar salários ou benefícios, mas também as empresas podem reduzir seus gastos. Estas questões são caracterizadas pelos autores como gerenciamento de resultados e que efetivamente não pode ser considerado como fraude contábil.

Para os gestores gerenciar os resultados de sua organização, Healy e Wahlen (1999) comentam que esse processo pode ser favorecido quando o gestor possui uma informação que o usuário externo não possui. Neste caso existe uma evidência explícita da existência de problemas de agência, que envolve o agente, que é o gestor; e o principal, sendo todos os stakeholders que possuam interesse na informação contábil.

Burgstahler e Dichev (1997), Healy e Wahlen (1999) e Martinez e Cardoso (2009) evidenciam que o gerenciamento dos resultados está correlacionado com a estratégia da empresa. Este fato é reforçado também por Jacob e Jorgensen (2007), ao analisarem os resultados fiscais de empresas norte americanas, cujos resultados da pesquisa confirmam os de Burgstahler e Dichev (1997), que empresas tendem a 
gerenciar seus ganhos e perdas para que suas metas ou objetivos do período sejam estabelecidos.

O que se pretende ao analisar estas obras, que descrevem o gerenciamento de resultados como consequência de decisões operacionais, é afirmar que a utilização da estratégia de ser social ou ambientalmente responsável pode ser uma forma de gerenciar os resultados (HALL; STAMMERJOHAN, 1997; HAN; WANG, 1998; CHIH; SHEN; KANG, 2008; PRIOR; SURRUCA; TRIBÓ, 2008). Isso justifica o pressuposto de Heltzer (2011), de que empresas responsáveis socialmente e/ou ambientalmente tendem a gerenciar mais seus resultados. Sendo as atividades ambientais das empresas uma das formas de aumentar seus gastos e consequentemente diminuir o resultado da empresa.

\subsection{Mensuração do Gerenciamento de Resultados}

Para o cálculo do índice de gerenciamento de resultados, Heltzer (2011) utiliza quatro formas diferentes de calculo. Três modelos estão baseados no modelo Jones e um trata-se do modelo original de Jones. O autor afirma adotar esta postura para que os resultados apresentem uma abrangência maior, e suas conclusões possam ser mais genéricas. Modelo Jones e suas versões modificadas são os mais utilizados em pesquisas internacionais, por serem mais eficientes na mensuração do gerenciamento de resultados (HELTZER, 2011 p. 80).

O primeiro modelo é o mais comum nas pesquisas internacionais e é modificado do modelo original de Jones. O autor trata esta sendo uma equação mais abrangente do que a original e não tão especifica do que as demais modelos apresentados na sequência.

Modelo 1

$\frac{\mathrm{TACC}_{\mathrm{i}}}{\mathrm{L} \_\mathrm{TA}_{\mathrm{i}}}=\alpha_{0}+\alpha_{1}\left(\frac{1}{\mathrm{~L}_{-} \mathrm{TA}_{\mathrm{i}}}\right)+\alpha_{2} \frac{\left(\Delta \mathrm{REV}_{\mathrm{i}}-\Delta \mathrm{AR}_{\mathrm{i}}\right)}{\mathrm{L}_{-} \mathrm{TA}_{\mathrm{i}}}+\alpha_{3} \frac{\mathrm{PPE}_{\mathrm{i}}}{\mathrm{L}_{-} \mathrm{TA}_{\mathrm{i}}}+\varepsilon_{\mathrm{j}}$

Onde:

TACC ${ }_{\mathrm{i}:}$ são os Accruals Totais; 
$L_{-} T A_{\mathrm{i}}$ : é o Ativo Total da empresa;

$\triangle R E V_{i}$ : é a variação da Receita;

$\triangle A R_{i}:$ é a variação das Contas a Receber;

$\mathrm{PPE}_{\mathrm{i}}$ : é o Imobilizado da empresa; e

$\varepsilon_{i}:$ é o índice de Gerenciamento de Resultados.

O segundo modelo adotado é o modelo original de Jones, que não considera a variável "variação das contas a receber".

Modelo 2

$\frac{\mathrm{TACC}_{\mathrm{i}}}{\mathrm{L}_{-} \mathrm{TA}_{\mathrm{i}}}=\alpha_{0}+\alpha_{1}\left(\frac{1}{\mathrm{~L}_{-} \mathrm{TA}_{\mathrm{i}}}\right)+\alpha_{2} \frac{\Delta \mathrm{REV}_{\mathrm{i}}}{\mathrm{L}_{-} \mathrm{TA}_{\mathrm{i}}}+\alpha_{3} \frac{\mathrm{PPE}_{\mathrm{i}}}{\mathrm{L}_{-} \mathrm{TA}_{\mathrm{i}}}+\varepsilon_{\mathrm{j}}$

A terceira equação toma a forma da primeira, entretanto adiciona as variáveis de BMi e CFO. A primeira é a razão entre Patrimônio Comum e Valor de Mercado das Ações Ordinárias, já a segunda é o Fluxo de Caixa Corrente. Este modelo foi proposto por Larcker e Richardson (2004).

Modelo 3

$\frac{\mathrm{TACC}_{\mathrm{i}}}{\mathrm{L}_{-} \mathrm{TA}_{\mathrm{i}}}=\alpha_{0}+\alpha_{1}\left(\frac{1}{\mathrm{~L}_{-} \mathrm{TA}}\right)+\alpha_{\mathrm{i}} \frac{\left(\Delta \mathrm{REV}_{\mathrm{i}}-\Delta \mathrm{AR}_{\mathrm{i}}\right)}{\mathrm{L}_{-} \mathrm{TA}_{\mathrm{i}}}+\alpha_{3} \frac{\mathrm{PPE}_{\mathrm{i}}}{\mathrm{L}_{-} \mathrm{TA}_{\mathrm{i}}}+\alpha_{4} \mathrm{BM}_{\mathrm{i}}+\alpha_{5} \frac{\mathrm{CFO}_{\mathrm{i}}}{\mathrm{L}_{-} \mathrm{TA}_{\mathrm{i}}}+\varepsilon_{\mathrm{j}}$

A quarta equação utilizada por Heltzer (2011) compreende o modelo descrito por Kothari et al. (2005). Este modelo adiciona a variável ROA, que significa Retorno sobre Ativo.

Modelo 4

$\frac{\mathrm{TACC}_{\mathrm{i}}}{\mathrm{L}_{-} \mathrm{TA}_{\mathrm{i}}}=\alpha_{0}+\alpha_{1}\left(\frac{1}{\mathrm{~L}_{-} \mathrm{TA}_{\mathrm{i}}}\right)+\alpha_{2} \frac{\left(\Delta \mathrm{REV}_{\mathrm{i}}-\Delta \mathrm{AR}_{\mathrm{i}}\right)}{\mathrm{L}_{-} \mathrm{TA}_{\mathrm{i}}}+\alpha_{3} \frac{\mathrm{PPE}_{\mathrm{i}}}{\mathrm{L}_{-} \mathrm{TA}_{\mathrm{i}}}+\alpha_{4} \mathrm{ROA}_{\mathrm{i}}+\varepsilon_{\mathrm{j}}$

O cálculo dos Accruals Totais é realizado por meio da diferença entre o Lucro

Revista Produção Online, Florianópolis, SC, v.14, n. 1, p. 143-170, jan./mar. 2014. 
Líquido e o Fluxo de Caixa Operacional. Assim os modelos utilizados descrevem que os accruals discricionários representados na equação por $\varepsilon_{j}$, são os resíduos da regressão linear para cada empresa. O valor dos accruals discricionários é descrito como o índice de gerenciamento de resultados da empresa.

\section{MÉTODO E PROCEDIMENTOS DA PESQUISA}

Este estudo pode ser classificado como uma pesquisa descritiva, pois procura expor a existência ou não de gerenciamento de resultados entre a adoção a responsabilidade social das empresas. De procedimentos documentais, pois utilizase das demonstrações contábeis do ano de 2010 e dos relatórios de responsabilidade social da BM\&FBovespa como fonte dos dados para a pesquisa. Além disso, com abordagem quantitativa, por utilizar-se de métodos matemáticos para obter seus principais resultados.

Como a proposta deste estudo foi de replicar o estudo de Heltzer (2011) em empresas brasileiras, selecionaram-se para esta amostra as empresas dos três níveis de governança corporativa da BM\&FBovespa, sendo eles Novo Mercado, Nível 1 e Nível 2. A escolha desta amostra decorre do fato de serem estes os segmentos que pressupõem maior transparência em suas demonstrações contábeis. O que se alinha também à pesquisa, pois esta investiga a questão do gerenciamento de resultados, e empresas pertencentes a esses segmentos devem possuir os objetivos dos gestores mais alinhados ao dos acionistas. A Tabela 1 traz a descrição da amostra utilizada para análise.

Tabela 1 - Característica do universo da amostra analisada

\begin{tabular}{llll}
\hline $\begin{array}{l}\text { Níveis de } \\
\text { Governança }\end{array}$ & $\begin{array}{l}\text { Total de } \\
\text { empresas }\end{array}$ & $\begin{array}{l}\text { Empresas com } \\
\text { responsabilidade } \\
\text { ambiental }\end{array}$ & $\begin{array}{l}\text { \% de empresas com } \\
\text { responsabilidade } \\
\text { ambiental }\end{array}$ \\
\hline Novo Mercado & 117 & 12 & $10,26 \%$ \\
Nível 1 & 38 & 8 & $21,05 \%$ \\
Nível 2 & 17 & 2 & $11,76 \%$ \\
Total & $\mathbf{1 7 2}$ & $\mathbf{2 2}$ & $\mathbf{1 2 , 7 9 \%}$ \\
\hline
\end{tabular}

Fonte: BM\&FBovespa. 
Com base na amostra, deu-se a coleta dos dados, inicialmente com as informações contábeis utilizadas para o cálculo do gerenciamento de resultados, sendo para tanto utilizado o Software Economática. Os dados coletados foram outrora descritos no item 3 e 4.1 deste artigo, onde se apresenta, respectivamente, o estudo de Heltzer (2011) e os modelos utilizados para a mensuração do gerenciamento de resultados.

Para analisar a atuação ambiental das empresas pertencentes à amostra, diferentemente do artigo de referência que utilizou a base de dados disponível KLD STATS, foram avaliados os projetos referentes à questão ambiental dispostos no portal eletrônico da BM\&FBovespa, que se dedica a evidenciar as atuações sociais e ambientais das empresas. A classificação se deu de forma dicotômica, sendo zero para quem não possui e um para quem possui. Foram considerados apenas os projetos realizados na área ambiental, que são classificados segundo a base de dados como sendo projetos ambientais e de mudanças climáticas.

Os itens avaliados foram os mesmos descritos na metodologia do artigo de Heltzer (2011), sendo seis itens para a força ambiental e sete itens para as preocupações ambientais. Utilizou-se, portanto, para a coleta de dados os mesmos critérios utilizados pelo artigo que se buscou replicar. Adicionalmente, cada informação utilizada no artigo citado foi utilizada também no presente artigo.

$\mathrm{Na}$ análise estatística aplicou-se o método de Regressão Linear Múltipla para a obtenção do índice de gerenciamento de resultados. E para verificar a existência de diferença estatística nas médias entre os grupos analisados foi utilizado o teste t. Os testes de médias foram considerados significantes a um coeficiente $\alpha=0,05$. Estes cálculos foram realizados no Software SPSS.

As limitações desta pesquisa se centram nos recortes efetuados para a elaboração do estudo, bem como pela utilização de uma metodologia idêntica ao estudo de Heltzer (2011). Assim os resultados não possibilitam a generalização, haja vista que se trabalhou com empresas listadas na BM\&FBovespa, desde que incluídas em algum dos segmentos de governança corporativa. Outra limitação é o fato desta pesquisa não considerar os motivos pelos quais as empresas desenvolvem atividades ambientais. 


\section{DESCRIÇÃO E ANÁLISE DOS RESULTADOS}

Para relacionar o gerenciamento de resultados com as atividades que descrevem a responsabilidade ambiental das empresas, de posse dos dados coletados na Economática, foi aplicado o método de Regressão Linear Múltipla. Este método consiste em descrever a relação entre as variáveis independentes (neste caso informações financeiras) e uma única variável dependente (neste estudo utilizou-se o valor dos accruals totais divididos pelo ativo total). A finalidade da utilização deste método está na obtenção do valor do resíduo de cada empresa para cada modelo, sendo que o resíduo descreve o valor do gerenciamento de resultados da empresa naquele ano.

A Tabela 2 descreve os resultados auferidos pelos quatro modelos analisados, contendo assim a informação de qual é a correlação entre variáveis independentes e dependentes, qual o índice de qualidade na correlação e, por fim, apresenta o coeficiente de significância do modelo. Cada modelo é considerado significante se obtiver seu $p$-value menor do que 0,05 .

Tabela 2 - Resultado das regressões dos modelos de gerenciamento de resultados

\begin{tabular}{llll}
\hline & $\mathbf{R}$ & R Square & Sig. \\
\hline Modelo 1 & 0,2852 & 0,0813 & 0,0032 \\
Modelo 2 & 0,2871 & 0,0824 & 0,0028 \\
Modelo 3 & 0,3331 & 0,1109 & 0,0020 \\
Modelo 4 & 0,2889 & 0,0835 & 0,0067 \\
\hline
\end{tabular}

Fonte: dados da pesquisa.

Os resultados descritos no modelo de análise de regressão apresentam um coeficiente de correlação de aproximadamente 0,30 para todos os quatro modelos, o que indica uma correlação de fraca para moderada. Entretanto, todos os modelos foram considerados significativos para serem analisados, podendo-se classificá-los como significantes a um nível de 1\%. Os resultados das regressões apontam para a possibilidade de auferir uma avaliação quanto ao nível de gerenciamento de resultados.

De posse das funções lineares obtidas por meio dos resultados descritos pela análise de regressão, calculou-se o resíduo da regressão para cada empresa que 
compôs a análise. Os resultados destes resíduos, que são os índices de gerenciamento de resultados, são descritos na Tabela 3.

Tabela 3 - Descrição dos valores de gerenciamento de resultados

\begin{tabular}{lllllll}
\hline Modelos & $\begin{array}{l}\text { Gerenciamento de } \\
\text { resultados }\end{array}$ & Média & Mediana & Mínimo & Máximo & $\begin{array}{l}\text { Desvio } \\
\text { Padrão }\end{array}$ \\
\hline \multirow{2}{*}{ Modelo 1 } & Gerenciamento & $-0,0006$ & $-0,0085$ & $-0,3936$ & 0,7232 & 0,1039 \\
& Gerenciamento Negativo & $-0,0602$ & $-0,0529$ & $-0,3936$ & $-0,0001$ & 0,0533 \\
& Gerenciamento Positivo & 0,0744 & 0,0475 & 0,0002 & 0,7232 & 0,1037 \\
Modelo 2 & Gerenciamento & 0,0000 & $-0,0088$ & $-0,3945$ & 0,7248 & 0,1038 \\
& Gerenciamento Negativo & $-0,0609$ & $-0,0565$ & $-0,3945$ & $-0,0006$ & 0,0530 \\
& Gerenciamento Positivo & 0,0739 & 0,0451 & 0,0003 & 0,7248 & 0,1033 \\
Modelo 3 & Gerenciamento & $-0,0003$ & $-0,0092$ & $-0,3821$ & 0,7223 & 0,1022 \\
& Gerenciamento Negativo & $-0,0560$ & $-0,0484$ & $-0,3821$ & $-0,0003$ & 0,0503 \\
& Gerenciamento Positivo & 0,0768 & 0,0450 & 0,0015 & 0,7223 & 0,1060 \\
Modelo 4 & Gerenciamento & 0,0000 & $-0,0093$ & $-0,3972$ & 0,7274 & 0,1038 \\
& Gerenciamento Negativo & $-0,0600$ & $-0,0539$ & $-0,3972$ & $-0,0002$ & 0,0526 \\
& NPositivo & 0,0736 & 0,0415 & 0,0003 & 0,7274 & 0,1039 \\
\hline
\end{tabular}

Fonte: dados da pesquisa.

A Tabela 3 evidencia o panorama dos resultados obtidos nos quatro modelos de gerenciamento de resultados. Para que a análise não fosse prejudicada, optou-se por trazer junto com o valor total do gerenciamento de resultados, os valores do gerenciamento positivo, e o valor do gerenciamento negativo. Esta opção é justificada, pois a média dos resíduos de uma regressão sempre tende a zero. Os resultados apontam para certa homogeneidade no índice de gerenciamento de resultados entre os quatro modelos, sendo que todos os valores descritos estão próximos das demais abordagens adotadas no estudo.

Após calculados os índices de gerenciamento de resultados, descrevem-se os resultados do teste de diferença de médias, realizados por meio de teste $-\mathrm{t}$. O teste - $t$, segundo Maroco (2007), necessita que sejam comprovados dois pressupostos, normalidade dos dados e homoscedasticidade. Foram realizados ambos os testes e para todas as amostras estes pressupostos se confirmaram. 


\subsection{Responsabilidade Ambiental versus Gerenciamento de Resultados}

Este tópico objetiva descrever os resultados do teste de diferença de médias, que responde a primeira questão descrita por Heltzer (2011): Empresas com responsabilidade ambiental exibem diferença estatisticamente significativa no gerenciamento de resultados se comparadas com empresas sem esta característica?

Tabela 4 - Teste de médias para a RQ1

\begin{tabular}{|c|c|c|c|c|c|}
\hline \multirow{2}{*}{ Modelos } & \multicolumn{2}{|c|}{ Teste de Levene } & \multicolumn{2}{|c|}{ Teste - $t$ de médias } & \multirow[b]{2}{*}{ Sig. (2-tailed) } \\
\hline & $\mathbf{F}$ & Sig. & $\mathbf{t}$ & df & \\
\hline Modelo 1 & 0,524606 & 0,469909 & $-2,64347$ & 165 & 0,00899714 \\
\hline Modelo 2 & 0,500282 & 0,480374 & $-2,66561$ & 165 & 0,00844882 \\
\hline Modelo 3 & 0,470375 & 0,493777 & $-2,53397$ & 165 & 0,01220933 \\
\hline Modelo 4 & 0,470855 & 0,493557 & $-2,71032$ & 165 & 0,00743246 \\
\hline
\end{tabular}

Fonte: dados da pesquisa.

A Tabela 4 aponta para os principais resultados obtidos no cálculo de diferença de médias dos quatro modelos do cálculo do gerenciamento de resultados. É possível perceber que o pressuposto de homoscedasticidade foi atendido, haja vista que o índice de significância foi superior a 0,05 , fundamentado no que afirma Maroco (2007). Considerando a diferença de médias é possível inferir que nos quatro modelos houve significância para a diferença entre as médias, tomando como significante todos os índices menores ou iguais a 0,05.

Tabela 5 - Discrição dos grupos envolvidos no teste de médias RQ1

\begin{tabular}{llllll}
\hline Modelos & Responsabilidade Ambiental & N & Média & Desvio Padrão & Erro Médio Padrão \\
\hline \multirow{2}{*}{ Modelo 1} & Com Responsabilidade & 21 & $-0,0556$ & 0,0911 & 0,0199 \\
& Sem Responsabilidade & 146 & 0,0073 & 0,1035 & 0,0085 \\
Modelo 2 & Com Responsabilidade & 21 & $-0,0554$ & 0,0915 & 0,0199 \\
& Sem Responsabilidade & 146 & 0,0079 & 0,1033 & 0,0085 \\
\multirow{2}{*}{ Modelo 3} & Com Responsabilidade & 21 & $-0,0523$ & 0,0894 & 0,0195 \\
& Sem Responsabilidade & 146 & 0,0071 & 0,1020 & 0,0084 \\
Modelo 4 & Com Responsabilidade & 21 & $-0,0563$ & 0,0918 & 0,0200 \\
& Sem Responsabilidade & 146 & 0,0081 & 0,1031 & 0,0085 \\
\hline
\end{tabular}

Fonte: dados da pesquisa. 
A Tabela 5 descreve que o gerenciamento de resultados em empresas com responsabilidade ambiental é de, em média, -0,05. Demonstra-se assim que empresas com responsabilidade ambiental tendem a reduzir seus resultados. Em valores absolutos é possível afirmar que empresas com esta característica gerenciam mais seus resultados. Desta forma a RQ1 é respondida, inferindo-se que empresas com responsabilidade ambiental gerenciam mais seus resultados.

\subsection{Força Ambiental versus Gerenciamento de Resultados}

Este tópico descreve os resultados do teste que responde a questão $R Q 2$ : Empresas com forças ambientais exibem diferença estatisticamente significativa no gerenciamento de resultados se comparadas com empresas sem esta característica? Para tanto foram comparadas as empresas que possuíam algum projeto voltado para as questões das forças ambientais com as empresas sem nenhum projeto.

Tabela 6 - Teste de médias para a RQ2

\begin{tabular}{llllll}
\hline Modelos & \multicolumn{4}{l}{ Teste de Levene } & \multicolumn{4}{l}{ Teste - T de médias } \\
\cline { 2 - 6 } & $\mathbf{F}$ & $\mathbf{S i g}$ & $\mathbf{t}$ & $\mathbf{d f}$ & Sig. (2-tailed) \\
Modelo 1 & 0,572354 & 0,450404 & $-2,23530$ & 165 & 0,026740 \\
Modelo 2 & 0,544564 & 0,461595 & $-2,25549$ & 165 & 0,025417 \\
Modelo 3 & 0,862353 & 0,354437 & $-2,02036$ & 165 & 0,044963 \\
Modelo 4 & 0,495350 & 0,482542 & $-2,30064$ & 165 & 0,022664 \\
\hline
\end{tabular}

Fonte: dados da pesquisa.

Os resultados descritos na Tabela 6 apontam para a existência de diferença estatística entre os índices de gerenciamento de resultados das empresas com e sem forças ambientais. Este fato se confirma, dado que para todos os quatro modelos os índices de significância se situaram abaixo de 0,05. É possível confirmar que a hipótese de homoscedasticidade foi aceita, segundo o que defende Maroco (2007). 
Tabela 7 - Discrição dos grupos envolvidos no teste de médias RQ2

\begin{tabular}{llllll}
\hline Modelos & Força Ambiental & N & Média & Desvio Padrão & Erro Médio Padrão \\
\hline \multirow{2}{*}{ Modelo 1 } & Com Força & 19 & $-0,0501$ & 0,0933 & 0,0214 \\
& Sem Força & 148 & 0,0058 & 0,1037 & 0,0085 \\
Modelo 2 & Com Força & 19 & $-0,0499$ & 0,0937 & 0,0215 \\
& Sem Força & 148 & 0,0064 & 0,1035 & 0,0085 \\
\multirow{2}{*}{ Modelo 3 } & Com Força & 19 & $-0,0445$ & 0,0905 & 0,0207 \\
& Sem Força & 148 & 0,0053 & 0,1025 & 0,0084 \\
Modelo 4 & Com Força & 19 & $-0,0509$ & 0,0941 & 0,0216 \\
& Sem Força & 148 & 0,0065 & 0,1034 & 0,0085 \\
\hline
\end{tabular}

Fonte: dados da pesquisa.

Dado o resultado que o teste de médias apresenta significância para os quatro modelos adotados, a Tabela 7 aponta as médias auferidas pelos grupos. Estes resultados estão em conformidade com os descritos no item 5.1, em que as médias do gerenciamento de resultados de empresas com projetos ambientais é de aproximadamente $-0,05$. O que indica que empresas com responsabilidade ambiental reduzem mais seus resultados.

\subsection{Preocupação Ambiental versus Gerenciamento de Resultados}

Este tópico se destina a responder a RQ3, que descreve a seguinte pergunta: Empresas com preocupação ambiental exibem diferença estatisticamente significativa no gerenciamento de resultados se comparadas com empresas que não possuem preocupação ambiental? De maneira semelhante ao executado no item 5.2, a análise comparou empresas com algum projeto que se enquadra dentre as categorias descritas por Heltzer (2011) com empresas sem nenhum destes projetos.

Tabela 8 - Teste de médias para RQ3

\begin{tabular}{llllll}
\hline Modelos & \multicolumn{2}{l}{ Teste de Levene } & \multicolumn{2}{l}{ Teste $-\mathbf{t}$ de médias } & Sig. (2-tailed) \\
& $\mathbf{F}$ & Sig. & $\mathbf{t}$ & df & 0,003618 \\
\hline Modelo 1 & 0,223301 & 0,637161 & $-2,95194$ & 165 & 0,003424 \\
Modelo 2 & 0,241117 & 0,624053 & $-2,96991$ & 165 & 0,005043 \\
Modelo 3 & 0,390363 & 0,532971 & $-2,84244$ & 165 & 0,003204 \\
Modelo 4 & 0,262997 & 0,608754 & $-2,99127$ & 165 &
\end{tabular}

Fonte: dados da pesquisa.

Os resultados do teste da questão RQ3 se assemelham com os obtidos nas 
questões RQ1 e RQ2. A Tabela 8 apresenta que, para todos os quatro modelos de gerenciamento de resultados, existem diferenças nos níveis de gerenciamento de resultados. O pressuposto de homoscedasticidade dos dados se confirma também para os quatro modelos, conforme ocorrido nos testes anteriores.

Tabela 9 - Discrição dos grupos envolvidos no teste de médias RQ3

\begin{tabular}{llllll}
\hline Modelos & Preocupação Ambiental & N & Média & Desvio Padrão & Erro Médio Padrão \\
\hline \multirow{2}{*}{ Modelo 1 } & Com Preocupação & 9 & $-0,0978$ & 0,1235 & 0,0411 \\
& Sem Preocupação & 158 & 0,0049 & 0,1003 & 0,0079 \\
\multirow{2}{*}{ Modelo 2 } & Com Preocupação & 9 & $-0,0976$ & 0,1241 & 0,0413 \\
& Sem Preocupação & 158 & 0,0055 & 0,1001 & 0,0079 \\
Modelo 3 & Com Preocupação & 9 & $-0,0925$ & 0,1220 & 0,0406 \\
& Sem Preocupação & 158 & 0,0049 & 0,0988 & 0,0078 \\
Modelo 4 & Com Preocupação & 9 & $-0,0983$ & 0,1249 & 0,0416 \\
& Sem Preocupação & 158 & 0,0055 & 0,1000 & 0,0079
\end{tabular}

Fonte: dados da pesquisa.

De forma análoga aos outros resultados, as médias das empresas com preocupação de resultados gerenciam seus resultados mais negativamente do que as demais. O que é comprovado pela média, do índice de gerenciamento de resultados, para os quatro modelos utilizados na pesquisa, ser de aproximadamente de $-0,09$.

\subsection{Maior força ambiental implica em maior gerenciamento de resultados}

Este item se destina a observar se o nível de gerenciamento de resultados é afetado com o aumento do número de projetos de força ambiental. Assim a análise objetiva responder a seguinte pergunta: As empresas com múltiplos níveis de forças ambientais exibem estatisticamente diferentes níveis de gerenciamento de resultados do que as empresas com uma única força ambiental? 
Tabela 10 - Teste de médias para RQ4

\begin{tabular}{llllll}
\hline Modelos & \multicolumn{2}{l}{ Teste de Levene } & \multicolumn{2}{l}{ Teste $-\mathbf{t}$ de médias } & \\
& $\mathbf{F}$ & Sig. & $\mathbf{t}$ & $\mathbf{d f}$ & Sig. (2-tailed) \\
\hline Modelo 1 & 1,41682 & 0,252425 & 0,804138 & 15 & 0,433877 \\
Modelo 2 & 1,395295 & 0,255903 & 0,800722 & 15 & 0,435792 \\
Modelo 3 & 1,758568 & 0,204643 & 0,824158 & 15 & 0,422762 \\
Modelo 4 & 1,42246 & 0,251524 & 0,798047 & 15 & 0,437296 \\
\hline
\end{tabular}

Fonte: dados da pesquisa.

Os testes de médias, descritos na Tabela 10, indicam que não existe diferença estaticamente comprovada para os níveis de gerenciamento de resultados, quando comparadas as duas subamostras. Este fato decorre do motivo que todos os índices de significância estão situados acima do valor de corte de 0,05. Porém, o teste de homoscedasticidade é atendido, conforme apresenta Maroco (2007).

Tabela 11 - Discrição dos grupos envolvidos no teste de médias RQ4

\begin{tabular}{llllll}
\hline Modelos & Responsabilidades & N & Média & Desvio Padrão & Erro Médio Padrão \\
\hline \multirow{2}{*}{ Modelo 1 } & Uma & 8 & $-0,0292$ & 0,0433 & 0,0153 \\
& Mais do que uma & 9 & $-0,0684$ & 0,1310 & 0,0437 \\
\multirow{2}{*}{ Modelo 2 } & Uma & 8 & $-0,0290$ & 0,0437 & 0,0155 \\
& Mais do que uma & 9 & $-0,0681$ & 0,1315 & 0,0438 \\
\multirow{2}{*}{ Modelo 3 } & Uma & 8 & $-0,0235$ & 0,0379 & 0,0134 \\
& Mais do que uma & 9 & $-0,0624$ & 0,1279 & 0,0426 \\
\multirow{2}{*}{ Modelo 4 } & Uma & 8 & $-0,0298$ & 0,0436 & 0,0154 \\
& Mais do que uma & 9 & $-0,0690$ & 0,1322 & 0,0441 \\
\hline
\end{tabular}

Fonte: dados da pesquisa.

Apesar de os testes de médias não apontarem para a existência de diferença estatística entre os níveis de gerenciamento de resultados, a Tabela 11 revela que empresas com uma única força ambiental gerenciam menos seus resultados. Entretanto, dada a não existência de significância, nenhuma avaliação pode ser feita de posse destes dados. 


\subsection{Mais preocupações ambientais implicam em maior gerenciamento de resultados}

Este tópico se destina a descrever os resultados auferidos no teste da questão RQ5, que se apresenta da seguinte forma: As empresas com múltiplos níveis de preocupações ambientais exibem estatisticamente diferentes níveis de gerenciamento de resultados do que as empresas com uma única preocupação ambiental? A análise desta questão se dá de forma análoga a questão RQ4.

Tabela 12 - Teste de médias para RQ5

\begin{tabular}{|c|c|c|c|c|c|}
\hline \multirow[t]{2}{*}{ Modelos } & \multicolumn{2}{|c|}{ Teste Levene } & \multicolumn{3}{|c|}{ Teste - t de médias } \\
\hline & $\mathbf{F}$ & Sig. & $\mathbf{t}$ & df & Sig. (2-tailed) \\
\hline Modelo 1 & 2,058064 & 0,19453 & 0,249878 & 7 & 0,809856 \\
\hline Modelo 2 & 2,028804 & 0,197359 & 0,254149 & 7 & 0,806684 \\
\hline Modelo 3 & 1,725449 & 0,230406 & 0,142258 & 7 & 0,890885 \\
\hline Modelo4 & 2,054584 & 0,194864 & 0,259403 & 7 & 0,802788 \\
\hline
\end{tabular}

Fonte: dados da pesquisa.

Os resultados apontados na Tabela 12 descrevem a não existência de relação entre o número de projetos ambientais com o nível de gerenciamento de resultados da empresa. Este fato é devido ao coeficiente de significância apresentado nos quatro testes ser em média de 0,8, que é superior ao nível de 0,05.

Tabela 13 - Discrição dos grupos envolvidos no teste de médias RQ5

\begin{tabular}{llllll}
\hline Modelos & Responsabilidades & $\mathbf{N}$ & Média & Desvio Padrão & Erro Médio Padrão \\
\hline \multirow{2}{*}{ Modelo 1 } & Uma & 4 & $-0,0856$ & 0,0424 & 0,0212 \\
& Mais do que uma & 5 & $-0,1076$ & 0,1701 & 0,0761 \\
\multirow{2}{*}{ Modelo 2 } & Uma & 4 & $-0,0852$ & 0,0429 & 0,0215 \\
\multirow{2}{*}{ Modelo 3 } & Mais do que uma & 5 & $-0,1077$ & 0,1708 & 0,0764 \\
\multirow{2}{*}{ Modelo 4 } & 4 & $-0,0857$ & 0,0482 & 0,0241 \\
& Mais do que uma & 5 & $-0,0981$ & 0,1673 & 0,0748 \\
& Uma & 4 & $-0,0855$ & 0,0425 & 0,0212 \\
& Mais do que uma & 5 & $-0,1086$ & 0,1719 & 0,0769 \\
\hline
\end{tabular}

Fonte: dados da pesquisa.

Observa-se na Tabela 13 que a média dos níveis de gerenciamento de resultados entre as subamostras comparadas é semelhante. O que corrobora com o resultado de médias apresentado na Tabela 12. Desta forma é possível inferir que o aumento das preocupações ambientais não implica no aumento dos níveis de 
gerenciamento de resultados.

\subsection{Análise e interpretação dos resultados}

Os resultados deste estudo, comparados com o estudo que o motivou, apresentam várias diferenças. Os testes de médias do estudo de Heltzer (2011) não apontam em nenhum momento para a utilização das atividades de responsabilidade ambiental como uma forma adotada pelos gestores para gerenciar seus resultados. O autor afirma que estas não passam de práticas já consolidadas junto às empresas e por este motivo não afetam, ou vão de encontro com o interesse dos proprietários ou acionistas.

O presente estudo obteve resultados diferentes na configuração da amostra. As questões RQ1, RQ2 e RQ3 apresentaram diferença estatística para o nível de gerenciamento de resultados. Indicam também que os gestores utilizam-se de atividades de responsabilidade ambiental para a redução do seu resultado, o que Martinez e Cardoso (2009) descrevem como uma forma de reduzir o percentual distribuído para os acionistas.

No que tange a afirmação de Heltzer (2011), de que em empresas norte americanas as práticas de responsabilidade ambiental são institucionalizadas, os resultados apresentados por este estudo sinalizam que as práticas podem estar institucionalizadas no Brasil. Entretanto as consequências destas atividades ambientais podem não ser claras para seus acionistas.

Os resultados descritos acima apontam para a direção descrita por Han e Wang (1998), que empresas de petróleo americanas se utilizam-se de atividades ambientais para melhorar sua visibilidade na comunidade em geral. Este paralelo se fundamenta no fato de que a maior parte das empresas que declararam seus projetos na BM\&FBovespa, são empresas que utilizam recursos naturais como fonte primaria de insumos para suas atividades.

Os resultados das questões RQ4 e RQ5 coadunam com a pesquisa de Heltzer (2011), que constatou a não existência de relação entre o número de atividades ambientais realizadas por uma empresa e o seu nível de gerenciamento 
de resultados.

A hipótese sustentada por Heltzer (2011), de que os gestores sem preocupação ambiental utilizam-se do gerenciamento de resultados para esconder ganhos desfavoráveis, não se confirmou neste estudo. Pois a média do gerenciamento de resultados em empresas com responsabilidade ambiental é maior do que a média das demais empresas. O que aponta para uma direção oposta à descrita pelo autor.

Os resultados desta pesquisa se assemelham mais com os achados de Hall e Stammerjohan (1997), Han e Wang (1998), Prior, Surruca e Tribó (2008) e Chih, Shen e Kang (2008), de que as atividades de responsabilidade social e ambiental das empresas são utilizadas pelos gestores como uma forma de gerenciamento de resultados por meio de decisões operacionais, do que os resultados apontados por Heltzer (2011). Este fato se justifica, pois os resultados mostram que empresas com responsabilidade ambiental gerenciam mais seus resultados. Entretanto, o aumento das atividades ambientais das empresas não implica em aumento do gerenciamento de resultados.

\section{CONCLUSÕES}

Este estudo objetivou analisar se empresas que possuem a característica de serem socioambientalmente responsáveis tendem a gerenciar mais seus resultados do que as sem essa característica. Assim, buscou-se descrever primeiramente qual é a relação existente entre o gerenciamento de resultados e as atividades de responsabilidade ambiental das empresas. Esta relação se justifica, pois como as atividades ambientais são decisões operacionais tomadas pelos gestores, elas podem interferir significativamente nos resultados das empresas. Este modelo de gerenciamento de resultados é tido como gerenciamento operacional, que não se enquadra como fraude contábil.

O presente estudo foi motivado pela pesquisa publicada de Heltzer (2011), por apresentar condições para reaplicação em outros cenários e com empresas de outra cultura organizacional, como é o caso das brasileiras. Além de apresentar este 
viés para os projetos de enfoque ambiental das empresas, que os autores admitem serem os gastos com a manutenção destes projetos, apresentam fatores determinantes para a redução do resultado das empresas. Neste caso, as evidencias brasileiras se mostraram diferentes das encontradas em empresas americanas, conforme a pesquisa de Heltzer (2011) naquele país.

Os testes estatísticos foram realizados com dados de empresas brasileiras pertencentes aos níveis de governança corporativa da BM\&FBovespa, e os resultados apontaram para a existência de diferença estatisticamente significante entre empresas com e sem responsabilidade ambiental. Estes achados sustentam a afirmação de que o desenvolvimento de atividades ambientais por parte dos gestores está acarretando redução nos resultados das empresas, o que vai de encontro com o interesse dos acionistas. Entretanto, os testes não apontaram a existência de correlação, nem positiva nem negativa, entre o número de projetos e o índice de gerenciamento de resultados.

Os resultados encontrados neste estudo são diferentes dos achados do estudo de Heltzer (2011), o que revela diferenças entre as empresas brasileiras e as empresas norte americanas. Isto sustenta a afirmação de que as atividades ambientais de empresas brasileiras estão afetando os resultados destas organizações, o que não foi observado no estudo de empresas norte-americanas. As empresas brasileiras estão com resultados menores por conta de gastos operacionais, que pode decorrer do desenvolvimento de atividades socioambientais. Entretanto não é possível afirmar que esta seja a única forma de aumento dos gastos, pois podem existir outros fatores na gestão que contribuíram para o gerenciamento dos resultados.

Desta forma, conclui-se que o objetivo de analisar se empresas que possuem a característica de serem socioambientalmente responsáveis tendem a gerenciar mais seus resultados do que as sem essa característica foi atendido, haja vista que os resultados dos testes empíricos descrevem diferença no gerenciamento de resultados de empresas com e sem responsabilidades ambientais. Sendo que empresas sem responsabilidade ambiental auferiram menor índice de gerenciamento de resultados que empresas com gerenciamento de resultados. $O$ 
que responde também a questão de pesquisa, para a confirmação de que as atividades ambientais aumentam o nível de gerenciamento de resultados.

Os resultados desta pesquisa e as limitações impostas à sua estratégia de operacionalização provocam inquietações, que podem ser objeto de investigação em futuras pesquisadas. Sugere-se utilizar segregação diferente para uma avaliação mais generalizada entre o gerenciamento de resultados e as atividades ambientais. Outra recomendação para pesquisas futuras é o questionamento aos gestores sobre os motivos que levam as empresas a adotarem atividades ambientalmente responsáveis.

\section{REFERÊNCIAS}

ASHLEY, P. A. Ética e responsabilidade social nos negócios. São Paulo: Saraiva, 2003.

BURGSTAHLER, D.; DICHEV, I. Earnings management to avoid earnings decreases and losses. Journal of Accounting and Economics, v. 24, , p. 99-126, 1997.

CARROLL, A. B. Corporate social responsibility. Business and Society, , v. 38, n. 3, p. 268-295, 1999.

$\mathrm{CHIH}, \mathrm{H} . ;$ SHEN, C.; KANG, F. Corporate social responsibility, investor protection, and earnings management: some international evidence. Journal of Business Ethics, v. 79, p. 179-198, 2008.

DONAIRE, D. Gestão ambiental na empresa. 2. ed. São Paulo: Atlas, 1995.

HALL, S.; STAMMERJOHAN, W. Damage awards and earnings management in the oil industry. The Accounting Review, v. 72, n. 1, p. 47-65, 1997.

HAN, J.; WANG, S. Political costs and earnings management of oil companies during the 1990 Persian Gulf Crisis, The Accounting Review, v. 73, n. 1, p. 103-117, 1998.

HEALY, P. M.; WAHLEN, J. M. A review of the earnings management literature and its implications for standard setting. Accounting Horizons, v. 13, n. 4, p. 365-384, 1999.

HELTZER, W. The asymmetric relationship between corporate environmental responsibility and earnings management: evidence from the United States. Managerial Auditing Journal, v. 26, n. 1, p. 65-88, 2011. 
HOLANDA, A. P.; CARDOSO, V. I. C.; OLIVEIRA, J. D.; DE LUCA, M. M. M. Investimentos em responsabilidade social: análise comparativa das empresas concessionárias de serviços públicos e não concessionárias listadas no ISE, da BM\&FBovespa. Pensar Contábil, v. 14, p. 26-34, 2012.

JACOB, J.; JORGENSEN, B. N. Earnings management and accounting income aggregation. Journal of Accounting and Economics, v. 43, p. 369-390, 2007.

LARCKER, D. F.; RICHARDSON, S. A. Fees paid to audit firms, accrual choices, and corporate governance, Journal of Accounting Research, v. 42, n. 3, p. 625-656, 2004.

MAROCO, João. Análise estatística com utilização do SPSS. 3. ed. rev. e corr. Lisboa: Sílabo, 2007.

MARTINEZ, A. L.; CARDOSO, R. L. Gerenciamento de resultados no Brasil mediante decisões operacionais. REAd. Revista Eletrônica de Administração, v. 15 , p. 1-27, 2009.

PRIOR, D.; SURROCA, J.; TRIBO, J. Are socially responsible managers really ethical? Exploring the relationship between earnings management and corporate social responsibility. Corporate Governance, v. 16, n. 3, p. 160-177, 2008.

RAUPP, F. M.; BEUREN, I. M. Caracterização da pesquisa em contabilidade. In: BEUREN, I. M. (Org.). Como elaborar trabalhos monográficos em contabilidade: teoria e prática. 2. ed. São Paulo: Atlas, 2004.

RIBEIRO, M. S.; BONFANTE, T. M.; GOMES, C. C. M. P.; CIOFFI, J. L.

Responsabilidade socioambiental no setor de papel e celulose. In: ENCONTRO DA ASSOCIAÇÃO NACIONAL DE PÓS-GRADUAÇÃO E PESQUISA EM ADMINISTRAÇÃO, 33., 2009, São Paulo-SP. Anais... Rio de Janeiro: ANPAD, 2009.

ROYCHOWDHURY, S.. Earnings management through real activities manipulation. Journal of Accounting and Economics, v. 42, p. 335-370, 2006.

SANTANA, N. B.; PÉRICO, A. E.; REBELATTO, D. A. N. Investimento em responsabilidade sócio-ambiental de empresas distribuidoras de energia elétrica: uma análise por envoltória de dados. Revista Gestão Industrial, v. 2, n. 4, p.124139, 2006.

SILVA, A.; LIMA, J. T. G. P.; CABRAL, A. C. A.; SANTOS, S. M.; PESSOA, M. N. M. Responsabilidade Social Empresarial: análise de relações entre a receita líquida e os investimentos socioambientais da Petrobras. Revista Produção Online, v. 13, n. 1, p. 108-133, 2013.

TACHIZAWA, T. Sustentabilidade e Responsabilidade Social: proposta de modelo de diagnóstico socioambiental baseada em pesquisa empírica. Revista Produção 
Online, v. 9, n. 4, p 795-821. 2009.

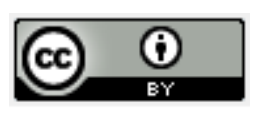

Artigo recebido em 24/03/2013 e aceito para publicação em 24/07/2013.

DOI: http://dx.doi.org/10.14488/1676-1901.v14.i1.1558

Revista Produção Online, Florianópolis, SC, v.14, n. 1, p. 143-170, jan./mar. 2014. 